\title{
Does adding hip exercises to quadriceps exercises result in superior outcomes in pain, function and quality of life for people with knee osteoarthritis? A systematic review and meta-analysis
}

\author{
Andrew Craig Hislop, ${ }^{1,2}$ Natalie J Collins \\ Adam Ivan Semciw (1) 2,4,5,6
}

- Additional material is published online only. To view, please visit the journal online (http://dx.doi.org/10.1136/ bjsports-2018-099683)

'Physiotherapy Department, Prince Charles Hospital, Australia

${ }^{2}$ School of Health and Rehabilitation Sciences, University of Queensland, Brisbane, Queensland, Australia ${ }^{3}$ School of Biomedical Sciences, University of Queensland, St Lucia, Queensland, Australia ${ }^{4}$ Physiotherapy Department, Princess Alexandra Hospital, Woolloongabba, Queensland, Australia

${ }^{5}$ Centre for Functioning and Health Research, Brisbane, Queensland, Australia ${ }^{6}$ Department of Rehabilitation, Nutrition and Sport, La Trobe University, Melbourne, Victoria, Australia

Correspondence to Dr Adam Ivan Semciw, Department of Rehabilitation, Nutrition and Sport, Latrobe University, Bundoora, VIC 3086, Australia;

a.semciw@latrobe.edu.au

Accepted 31 December 2018 Published Online First 6 February 2019 Chermside, Queensland,

\section{ABSTRACT \\ Objectives To determine, in people with knee} osteoarthritis (KOA): i) the effectiveness of adding hip strengthening exercises to quadriceps exercises and ii) the type of hip strengthening exercise with the greatest evidence for improving pain, function and quality of life. Design Systematic review with meta-analysis.

Data sources Medline, Embase, Cochrane, CINAHL and SportDiscus databases were searched from inception to January 2018.

\section{Eligibility criteria for selecting}

studies Randomised controlled trials investigating the effect of adding hip exercises to quadriceps exercises in people with KOA on pain, function and/or quality of life were included. Three subgroups of hip exercises were included: resistance, functional neuromuscular or multimodal exercise.

Results Eight studies were included. Pooled data provide evidence that combined hip and quadriceps exercise is significantly more effective than quadriceps exercise alone for improving walking function (standardised mean difference $-1.06,95 \% \mathrm{Cl}-2.01$ to -0.12$)$, but not for outcomes of pain $(-0.09$, $95 \% \mathrm{Cl}-0.96$ to 0.79$)$, patient-reported function $(-0.74,95 \% \mathrm{Cl}-1.56$ to 0.08$)$ or stair function (-0.7, $95 \% \mathrm{Cl}-1.67$ to 0.26$)$. Subgroup analyses reveal that hip resistance exercises are more effective than functional neuromuscular exercises for improving pain $(p<0.0001)$ and patient-reported function $(p<0.0001)$. Multimodal exercise is no more effective than quadriceps strengthening alone for pain $(0.13,95 \% \mathrm{Cl}-0.31$ to $0.56)$, patient-reported function $(-0.15,95 \% \mathrm{Cl}-0.58$ to 0.29$)$ or stair function $(0.13,95 \% \mathrm{Cl}-0.3$ to 0.57$)$. Conclusion Walking improved after the addition of hip strengthening to quadriceps strengthening in people with KOA. The addition of resistance hip exercises to quadriceps resulted in greater improvements in patientreported pain and function.

Check for updates

(C) Author(s) (or their employer(s)) 2020. No commercial re-use. See rights and permissions. Published by BMJ.

To cite: Hislop AC

Collins NJ, Tucker $\mathrm{K}$,

et al. Br J Sports Med

2020:54:263-271.

\section{INTRODUCTION}

Knee osteoarthritis (KOA) is a heterogenous disease that can affect bone, cartilage, synovium and meniscii. ${ }^{1}$ It results in pain, stiffness and a reduced ability to perform activities of daily living. ${ }^{2}$ One in four people aged over 55 years are affected by symptomatic $\mathrm{KOA}^{4}{ }^{4}$ making it the leading cause of pain and disability among adults. ${ }^{245}$ Due to an ageing population and increasing obesity, the prevalence of $\mathrm{KOA}$ is expected to rise, increasing the burden of disease on health services globally. ${ }^{6}$ Interventions that improve symptoms and reduce prevalence through primary and secondary prevention programmes are paramount to minimise the escalating social and personal costs of KOA. ${ }^{56}$

Land-based exercise and strength training are recommended as core treatments for all individuals with $\mathrm{KOA}^{7}$ A Cochrane review of 44 trials (3537 participants) demonstrated that land-based exercise reduced pain and improved function in people with KOA over the short-term, with a large effect size, when compared with a non-exercise intervention or wait-and-see. ${ }^{2}$ Recent systematic reviews have demonstrated greater gains in quadriceps strength with high-intensity compared with low-intensity resistance training in people with $\mathrm{KOA}^{8}{ }^{8}$ and that gains in quadriceps strength are associated with greater improvements in symptoms and disability. ${ }^{9}$ Quadriceps strength training, however, is not superior to other types of exercise (eg, neuromuscular or aerobic exercise). ${ }^{9}$ Further research is needed to identify the optimal content of exercise programmes for people with KOA. ${ }^{2}$

A recent systematic review and meta-analysis identified moderate quality evidence that hip abduction strength is $7 \%-24 \%$ weaker in people with KOA compared with age-matched controls. ${ }^{10}$ Hip abduction strength is also significantly related to both physical ${ }^{11}$ and self-reported function ${ }^{12}$ in people with KOA. The biomechanical role of the hip abductors is primarily to maintain frontal plane stability during stance phase. ${ }^{11}$ For example, variance in hip strength between individuals with KOA is highly related to peak hip adduction angles and contralateral pelvic drop. ${ }^{12}$ This pelvic drop associates to an increase in knee adduction moment in a healthy population. ${ }^{13}$ The function of the hip abductors has also been implicated in KOA progression. In those with less severe KOA, it has been suggested that greater hip abduction strength is able to maintain altered positions of the trunk, and results in similar first and reduced second peak knee adduction moments compared with matched controls. ${ }^{14}$ This gait adaptation controlling medial joint load may explain why stronger hip abduction muscles have a protective effect against KOA progression over 18 months. ${ }^{15}$

Hip strengthening alone and in conjunction with quadriceps strengthening is recommended, ${ }^{16}$ based 
on studies that have compared these with non-exercise interventions. ${ }^{17}{ }^{18}$ However, there is conflicting evidence in clinical outcomes when supplementing quadriceps exercises with hip exercises. ${ }^{19} 20$ There is a need to quantitatively synthesise the evidence to provide clinical recommendations regarding exercise prescription for KOA.

The aims of this systematic review and meta-analysis were to: (i) determine the effectiveness of adding hip exercises to a quadriceps exercise programme on pain, function and quality of life (QoL) in people with symptomatic KOA and (ii) determine what type of hip exercise (eg, resistance, functional neuromuscular, multimodal) has the greatest evidence for improving pain, function and QoL in people with KOA.

\section{METHODS}

This systematic review was prospectively registered on PROSPERO (CRD42017057857).

\section{Search strategy}

Medline, Embase, Cochrane, CINAHL and SportDiscus databases were searched from inception to January 2018, using a comprehensive search strategy (online supplementary file 1). Search terms were modified for each database. The search strategy was based on two broad concepts: (i) KOA and (ii) exercise. Medical subject headings were searched by title and abstract for each concept and combined with the 'OR' Boolean operator. The two concepts were then combined with the 'AND' Boolean operator. Results from each database were exported to Endnote X7 (Thomson Reuters, California, USA) and duplicates removed.

All articles were screened for inclusion by two independent reviewers (ACH, MD) according to title and abstract, using specific eligibility criteria. Discrepancies were resolved by a third reviewer (AIS). Full texts of remaining articles were obtained and screened by two reviewers (ACH, MD). Citation tracking and reference checking of included articles were completed by one reviewer $(\mathrm{ACH})$ using Google Scholar.

\section{Study selection criteria}

Randomised controlled trials (RCTs) were included if outcomes were reported in a peer-reviewed journal. Non-English studies were excluded. Studies were included based on the criteria discussed below.

\section{Population}

People with symptomatic primary tibiofemoral KOA (radiographically confirmed) in either one or both knee(s).

\section{Intervention}

Land-based strengthening exercises that targeted muscles with an action at the hip joint, in addition to quadriceps exercises.

The type and dosage of hip exercises described in each study were categorised as either: (i) high-intensity resistance; (ii) low-intensity resistance; (iii) functional neuromuscular or (iv) multimodal exercise (table 1). Resistance training was characterised by techniques that included weight training ${ }^{20-22}$ or elastic devices ${ }^{19}$ that target the hip musculature ${ }^{8}$ (eg, elastic band-resisted abduction during side-stepping). ${ }^{19}$ Functional neuromuscular exercises ${ }^{23-25}$ were defined by the execution of a functional movement requiring single-leg demand, given that single-leg demand is associated with gluteal muscle activity ${ }^{26}$ (eg, stepping ${ }^{1924}$ or step-up/step-down). ${ }^{192325}$ Multimodal exercise ${ }^{19}$
Table 1 Types and definition of hip strengthening exercises

\begin{tabular}{ll}
\hline Type of exercise & Definition of exercise \\
\hline $\begin{array}{l}\text { High-intensity resistance } \\
\text { strength training }\end{array}$ & $\begin{array}{l}\geq 70 \% 1 \mathrm{RM} \text { and multiple sets of repetitions } \\
\text { with }<12 \text { repeats per set to fatigue }\end{array}$ \\
$\begin{array}{l}\text { Low-intensity resistance } \\
\text { strength training }\end{array}$ & $<70 \% 1 \mathrm{RM}$ or repetitions $\geq 12^{8}$ \\
$\begin{array}{l}\text { Functional neuromuscular } \\
\text { exercise }\end{array}$ & $\begin{array}{l}\text { A functional movement demanding single leg stance (eg, } \\
\text { stepping, step-ups, single-leg squat, step-downs) }\end{array}$ \\
\hline $\begin{array}{l}\text { Multimodal exercise } \\
\text { Combination of resistance strength training (high- } \\
\text { intensity or low-intensity) and functional neuromuscular } \\
\text { exercise }\end{array}$ \\
\hline
\end{tabular}

RM, repetition maximum.

was used to categorise interventions that included a combination of resistance and functional neuromuscular exercises.

\section{Comparison}

The comparator intervention was quadriceps strengthening exercises. Studies that used a usual care or wait-list comparator were not included (deviating from original PROSPERO registration), as quadriceps strengthening is considered current best practice. ${ }^{27}$ We also excluded studies that used non-exercise-based interventions as a comparator (eg, weight loss, orthoses, injections).

\section{Outcomes}

Measures included pain, self-reported physical function, physical function tests or QoL. Where studies included more than one measurement tool, effect sizes were calculated using the tool highest on a suggested hierarchy of continuous outcomes (online supplementary file 2). ${ }^{28}$ Physical function tests were selected based on the Osteoarthritis Research Society International (OARSI) recommended set of performance-based measures of physical function. ${ }^{29}$

\section{Data extraction}

Data extracted from studies included number of participants, participant characteristics (age, gender, body mass index) ${ }^{30}$ and detailed descriptions of the intervention programmes as per the Consensus on Exercise Reporting Template (CERT). ${ }^{31}$ Post-test means and SD were collected or imputed from available information. ${ }^{32}$ Where sufficient data were unavailable, or could not be imputed, corresponding authors were contacted for further information. Data were extracted by one reviewer $(\mathrm{ACH})$ and checked by a second reviewer (AIS).

\section{Assessment of risk of bias}

We used the Physiotherapy Evidence Database (PEDro) scale to assess risk of bias of each included RCT. The PEDro scale has fair to good inter-rater reliability, ${ }^{33}$ and is a valid measure of methodological quality for controlled trials. ${ }^{34}$ This scale comprises 11 items which identify the presence or absence of key methodological components of clinical trials and provides an overall score out of 10 . Scores were taken directly from those archived in the PEDro database where available, otherwise studies were assessed independently by two reviewers (ACH and MD). Discrepancies were resolved by consensus with a third reviewer (AIS). Studies were dichotomised as high-quality (PEDro score $>6$ ) or lowquality (score $\leq 6$ ) using established methods. ${ }^{35}$ No studies were excluded based on quality appraisal. 


\section{Data synthesis}

Studies were grouped according to outcome measures and postintervention assessment time points. Time points were defined as 'short-term' ( $\leq 3$ months), 'medium-term' (3-12 months) and 'long-term' (>12 months). ${ }^{36}$ Postintervention means, SDs and sample size were entered into Review Manager V.5.3 (RevMan) to calculate standardised mean differences (SMDs) with 95\% CI for each outcome measure. Data were pooled in a meta-analysis (random effects) where studies reported similar outcomes and time points. Separate subgroup analyses were performed according to type of hip intervention (high-intensity resistance, low-intensity resistance, functional neuromuscular or multimodal; $\mathrm{p}<0.05$ indicates a statistically significant difference across exercise types) or confirmed medial tibiofemoral compartment involvement. Sensitivity analyses were conducted including only high-quality studies. Effect sizes of $0.2,0.5$ and 0.8 were considered small, moderate and large, respectively. ${ }^{37}$ Statistical heterogeneity was assessed using the $\mathrm{I}^{2}$ statistic. Values of $25 \%, 50 \%$ and $75 \%$ were considered low, moderate and high levels of heterogeneity, respectively. ${ }^{38}$

For each meta-analysis, the quality of the body of evidence was evaluated using a modified version of the Grading of Recommendations Assessment, Development and Evaluation (GRADE) approach. ${ }^{39}$ This grades the quality of evidence as high, moderate, low or very low across four domains: risk of bias, inconsistency, indirectness and imprecision. ${ }^{40}$ The evidence for each meta-analysis was initially considered high and was downgraded one level (moderate, low, very low) for each item that was not satisfied: risk of bias (majority of studies low PEDro score $\leq 6)$, inconsistency $\left(\mathrm{I}^{2} \geq 50 \%\right),{ }^{10}$ indirectness (clinically heterogeneous, eg, differences in included KOA severity or knee compartment affected) and imprecision (upper or lower CI $>0.5$ in either direction). ${ }^{10}$ When no meta-analysis was possible, the level of evidence was described as limited.

\section{RESULTS}

Article identification and selection

Identification and selection of articles is summarised in figure 1. The search strategy yielded 10018 articles (after duplicates were removed). After screening of titles and abstracts, 411 full-text articles were assessed for eligibility, and 8 studies were included.

\section{Study characteristics}

Characteristics and outcomes for each study are summarised in table 2 . A total of 341 participants (34\% male) were included. One study ${ }^{20}$ did not report participant sex. Only three studies ${ }^{19} 2425$ reported body mass index (BMI), with mean BMI ranging from 25 to $31 \mathrm{~kg} / \mathrm{m}^{22425}$ across $60 \%$ of the total participant pool. Two studies included participants with pain severity $\geq 25 \mathrm{~mm}^{19}$ or $\geq 30 \mathrm{~mm}^{22}$ on $100 \mathrm{~mm}$ visual analogue scale (VAS) (totalling 130 participants). Four studies ${ }^{19-22}$ (56\% of all participants) included participants with medial compartment KOA. KellgrenLawrence severity ranged from grade $1^{21}$ to $4^{19}$ in the five studies that reported radiographic severity. ${ }^{19} 21-2325$

A summary of exercises, time points and outcomes are presented in table 3 . One study used high-intensity ${ }^{21}$ and two studies used low-intensity hip resistance exercise. ${ }^{20} 22$ The multimodal exercise programme combined a low-intensity hip resistance exercise with functional neuromuscular exercise. ${ }^{19}$ High-intensity quadriceps resistance exercises were used in one study of each of the three subgroups of hip exercise (low-intensity

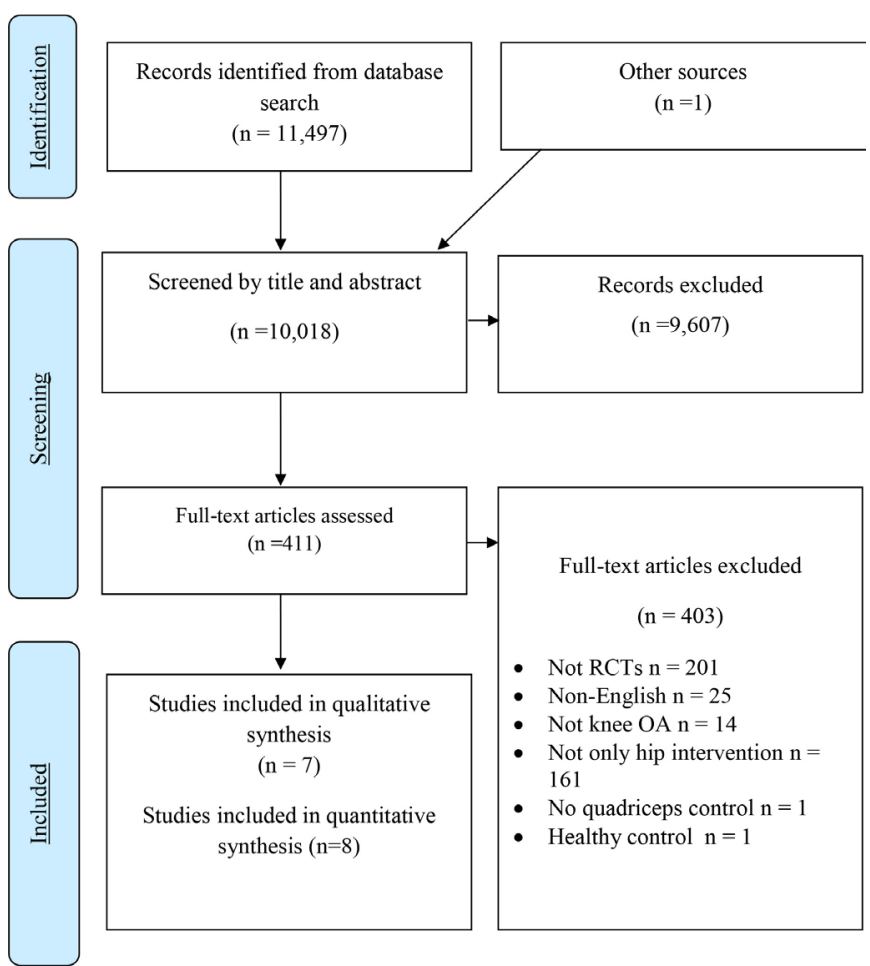

Figure 1 Preferred Reporting Items for Systematic Reviews and MetaAnalyses flow diagram. OA, osteoarthritis; RCT, randomised controlled trial.

resistance hip exercise ${ }^{20}$; functional neuromuscular ${ }^{25}$; multimodal programme ${ }^{19}$ ). One study of a functional neuromuscular programme ${ }^{23}$ included low-intensity quadriceps strengthening in the intervention but high-intensity quadriceps strengthening in the control group.

Interventions and assessment time points varied from $4^{2441}$ to 12 weeks. ${ }^{19} 25$ No studies evaluated medium-term or long-term outcomes. Six studies ${ }^{19-21232541}$ measured global pain on a VAS, and one study ${ }^{24}$ used the Knee Injury and Osteoarthritis Outcome Score (KOOS) pain subscale. Patient-reported function was measured with the Western Ontario and McMaster Universities Osteoarthritis Index in four studies, ${ }^{19-22}$ and the KOOS-activities of daily living in one study. ${ }^{24}$ Five studies ${ }^{192022-24}$ reported on at least one of the OARSI recommended performance-based tests of physical function. ${ }^{42}$ Two studies ${ }^{19}{ }^{20}$ reported balance outcomes using a step test. Two studies reported on QoL outcomes. One study evaluated health-related QoL using the assessment of quality of life scale, ${ }^{19}$ and one study evaluated knee-related QoL using KOOS-QoL. ${ }^{24}$

Reporting of interventions within each study is summarised in the CERT figure in online supplementary file 3. Type of exercise equipment, details of exercise individualisation and whether it was a tailored or generic exercise programme were all well reported across studies. Instructor qualifications, motivation strategies, adverse events, programme fidelity and adherence were poorly reported.

\section{Assessment of risk of bias and quality of evidence}

Risk of bias scores (PEDro) for each study are reported in online supplementary file 4 . The mean PEDro score was 6 (range $4^{41}$ to $7^{1924}$ ). Two studies were rated as high-quality ${ }^{19} 24$ and six studies as low-quality. ${ }^{20-232541}$ Due to the nature of exercise therapy, none of the studies were able to meet criteria for blinding of 
Table 2 Characteristics of included studies

\begin{tabular}{|c|c|c|c|c|c|c|c|c|c|}
\hline & \multicolumn{5}{|c|}{ Participant characteristics } & \multicolumn{4}{|c|}{ Outcome measures } \\
\hline & KOA diagnosis & $\begin{array}{l}\mathrm{N} \text { total ( } \mathrm{N} \\
\text { males) }\end{array}$ & Age (years) & $\begin{array}{l}\text { BMI }(\mathrm{kg} / \\
\left.\mathrm{m}^{2}\right)\end{array}$ & $\begin{array}{l}\mathrm{K}-\mathrm{L} \\
\text { grade }\end{array}$ & Pain & $\begin{array}{l}\text { Patient- } \\
\text { reported } \\
\text { function }\end{array}$ & Physical function tests & QoL \\
\hline Bennell et $a l^{19}$ & MC, pain $\geq 25 / 100$ & $100(48)$ & $62.7(7.3)$ & $\begin{array}{l}29.7 \\
(3.9)\end{array}$ & $2-4$ & Global, walking & WOMAC & $\begin{array}{l}10 \mathrm{~m} \text { walk } \\
\text { Stair negotiation } \\
30 \text { s STS; step test } \\
\text { Square step test } \\
\text { Single leg balance }\end{array}$ & AQoL \\
\hline Callaghan et a $/^{41}$ & ACR & $18(12)$ & 59 (range 35-80) & NR & NR & Global & & $50 \mathrm{~m}$ walk & \\
\hline $\begin{array}{l}\text { Chaipinyo and } \\
\text { Karoonsupcharoen } \\
24\end{array}$ & ACR & $48(11)$ & $62(6)$ & $\begin{array}{l}25 \\
\text { (4) }\end{array}$ & NR & KOOS-pain & KOOS-PS & $\begin{array}{l}50 \mathrm{~m} \text { walk; } 15 \mathrm{~m} \text { walk } \\
\text { TUG/GUAG } \\
\text { Stair negotiation }\end{array}$ & KOOS-QoL \\
\hline Olagbegi et al ${ }^{25}$ & $A C R$ & 55 (4) & $61.1(13.75)$ & $\begin{array}{l}31.26 \\
(8.54)\end{array}$ & 2 only & Global, walking & PASE & & \\
\hline Verma et $a l^{20}$ & $\mathrm{U}, \mathrm{MC}$ & 30 (NR) & $50-70$ & NR & NR & Global & WOMAC & $\begin{array}{l}\text { TUG/GUAG } \\
\text { Step test }\end{array}$ & \\
\hline
\end{tabular}

Pain outcome measures: global pain measured on visual analogue scale; KOOS-pain; KPS.

Patient-reported function: WOMAC; KOOS-PS; PASE.

Physical function tests: 30 s STS; TUG/GUAG; 6MWT.

Diagnosis: ACR meets clinical criteria for classification and reporting of KOA.

ACR, American College of Rheumatology; B, bilateral; U, unilateral; MC, medial compartment; 6MWT, 6 min walk test; KOA, knee osteoarthritis; KOOS-pain, Knee injury and Osteoarthritis Outcome Score pain subscale; KPS, Knee Pain Score; KOOS-PS, Knee Osteoarthritis Outcome Score; NR, not reported; PASE, Physical Activity Scale for the Elderly; K-L, Kellgren-Lawrence; QoL, quality of life; AQoL, assessment of quality of life; STS, sit to stand test; TUG/GUAG, timed up and go, get up and go; WOMAC, Western Ontario and McMaster Universities Osteoarthritis Index; NR, not reported.

participants or therapists, while four studies incorporated assessor blinding. Four studies reported intention-to-treat analysis. ${ }^{17202125}$ Three studies did not meet criteria for adequate follow-up. 192541

\section{Patient-reported outcomes}

Pain

Seven studies (two high-quality ${ }^{19} 24$ and five lowquality $^{20} 21232541$ ) totalling 311 participants (157 intervention; 154 control) presented outcomes of patient-reported pain (figure 2A). Five studies ${ }^{19-212425}$ provided sufficient data to be pooled in a meta-analysis, irrespective of exercise type. Pooled findings demonstrated that combining hip and quadriceps exercises was no more effective than quadriceps exercises alone in reducing pain (SMD $-0.09,95 \% \mathrm{CI}-0.96$ to 0.79 ). Outcomes were not different when including only those with medial KOA $(0.73,95 \% \mathrm{CI}-1.74$ to 0.28 ) (online supplementary file 6$) .{ }^{19-21}$

Tests of subgroup differences demonstrated a significant difference between types of hip exercises employed $(p<0.01)$ (figure 2A). Data from low-quality single studies provide limited evidence that high-intensity $(-1.18,95 \% \mathrm{CI}-1.97 \text { to }-0.4)^{21}$ and low-intensity $(-1.26,95 \% \mathrm{CI}-2.05 \text { to }-0.47)^{20}$ resistance hip exercise in combination with quadriceps exercises were superior to quadriceps alone. Functional neuromuscular hip exercises had no additional benefit to quadriceps exercises alone $(0.86$, $95 \%$ CI -0.13 to 1.85 ). Two additional low-quality studies ${ }^{23} 41$ that evaluated functional neuromuscular hip exercises had insufficient data to calculate effect sizes. These studies also reported no additional benefit of neuromuscular hip exercises to quadriceps alone. ${ }^{23}{ }^{41}$ Data from a single high-quality study ${ }^{19}$ demonstrated no differences between multimodal hip and quadriceps exercises, versus quadriceps exercises alone $(0.13,95 \%$ CI -0.31 to 0.56$)$.

\section{Function}

Five studies (two high-quality ${ }^{19} 24$ and three low-quality ${ }^{20-22}$ ) totalling 238 participants evaluated outcomes of patient-reported function. Pooled data suggest no significant difference between hip and quadriceps exercises combined and quadriceps exercises alone $(-0.74,95 \% \mathrm{CI}-1.56$ to 0.08$)$ (figure $2 \mathrm{~B}$ ). When stratified by medial KOA, pooled data from four studies ${ }^{19-22}$ showed that hip and quadriceps exercises was significantly more effective than quadriceps alone $(-0.73,95 \%$ CI -1.91 to -0.21$)$ (online supplementary file 6).

Subgroup analyses revealed that the type of hip exercise may influence the potential benefit of adding hip exercise to quadriceps exercise in KOA (test for subgroup differences, $\mathrm{p}<0.001$ ). Adding resistance hip exercises to quadriceps exercises appears to have added benefit on patient-reported function compared with quadriceps exercises alone. Two studies of low-intensity resistance exercises provided evidence of large effect $(-1.25$, $95 \%$ CI -2.11 to -0.39$),{ }^{20} 22$ while data from a high-intensity single study provided limited evidence of a large effect $(-1.77$, $95 \%$ CI -2.63 to -0.90$).{ }^{21}$ There was no significant effect of adding functional neuromuscular or multimodal exercises to quadriceps exercises alone, based on findings of one study $(0.52$, $95 \%$ CI -0.10 to 1.14$){ }^{19}$

\section{Quality of life}

QoL was reported in two high-quality studies, ${ }^{19}{ }^{24}$ totalling 148 participants. Outcomes of knee-related QoL and health-related 
Table 3 Interventions for both intervention and control groups, duration and results

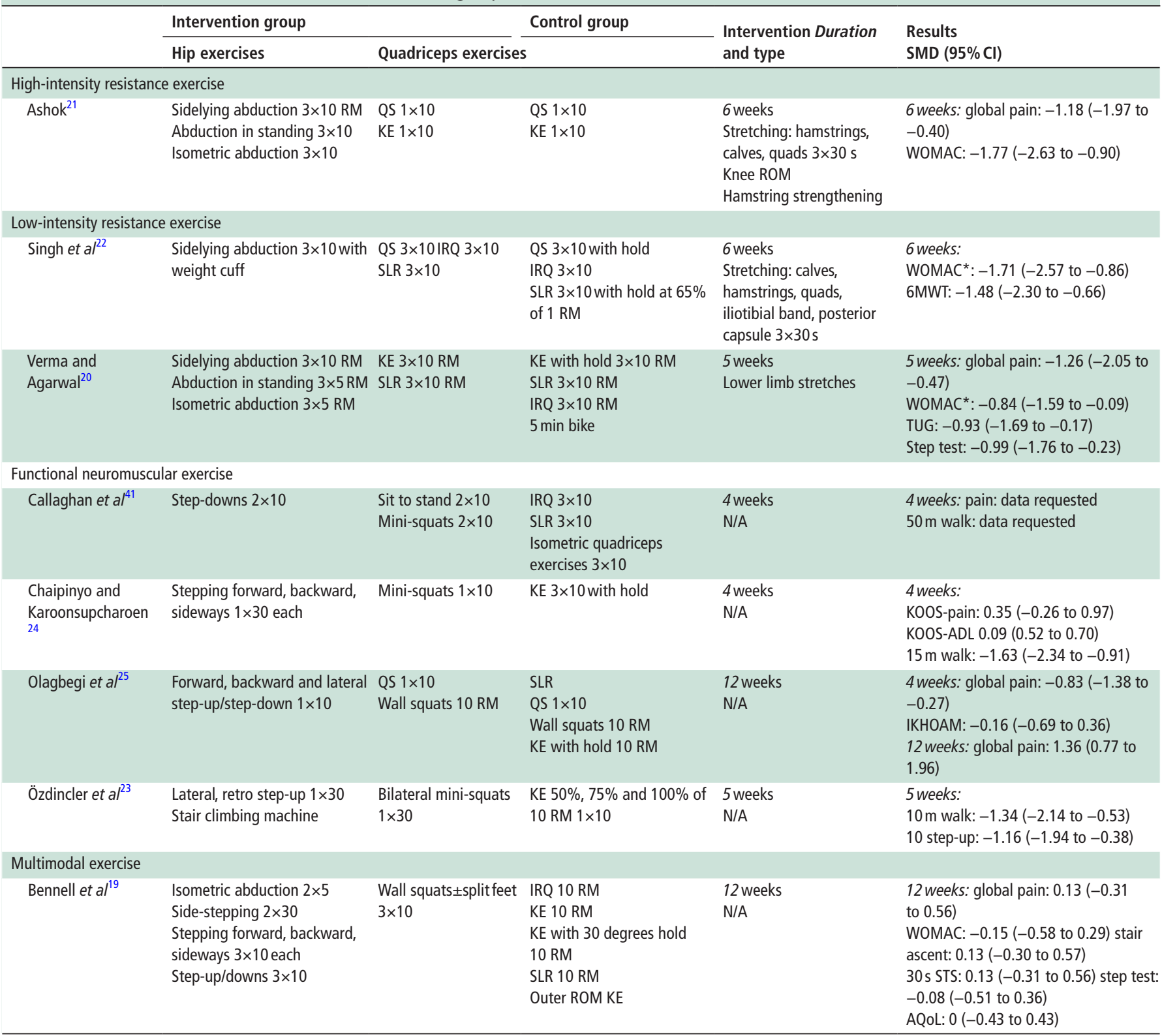

Global pain/pain was assessed using VAS.

${ }^{*}$ All WOMAC subscales.

$3 \times 10 / 5$ RM, 3 repetitions of 10/5 repetition maximum; QS, quadriceps setting; IRQ, inner range quadriceps; SLR, straight leg raise; KE, knee extension; VAS, visual analogue scale; WOMAC, Western Ontario and McMaster Universities Osteoarthritis Index; KOOS, Knee injury and Osteoarthritis Outcome Score; N/A, not available; ADL, activities of daily living; IKHOAM, Ibadan Knee/Hip Osteoarthritis Outcome Measure; 6MWT, 6 min walk test; TUG, timed up and go.

QoL were not pooled as they measure different constructs. ${ }^{43}$ Limited evidence from single studies demonstrated that neither the functional neuromuscular hip exercise programme $(-0.43$, $95 \% \mathrm{CI}-1.05$ to 0.19$)^{24}$ or multimodal hip exercise programme $(0.00,95 \%$ CI -0.43 to 0.43$),{ }^{19}$ when combined with quadriceps exercises, were significantly more effective than a quadriceps only programme in improving QoL (figure 2C).

\section{Performance-based measures of physical function}

A total of 256 participants across six studies (two high-quality ${ }^{1924}$ and four low-quality $2022{ }^{41}$ ) presented at least one outcome corresponding to the five recommended activities by OARSI (table 2). ${ }^{29}$

\section{Walking}

Five studies (two high-quality ${ }^{19} 24$ and three low-quality ${ }^{22} 2341$ ) totalling 226 participants presented outcomes for walking. Data from four studies were pooled in a meta-analysis with a large effect size favouring combined hip and quadriceps exercise over quadriceps exercise alone $(-1.06,95 \% \mathrm{CI}-2.01$ to -0.12$)$ (figure 3A). When only medial KOA studies ${ }^{19} 22$ were included in meta-analyses, the addition of hip exercises to quadriceps was no different to quadriceps alone $(-0.68,95 \% \mathrm{CI}-2.17$ to 0.82$)$ (online supplementary file 7).

Subgroup analyses demonstrated a significant difference between types of hip exercises (test for subgroup differences, $\mathrm{p}<0.001)$. No studies investigated the effects of high-intensity hip resistance exercises on walking function. Large effect sizes 
A

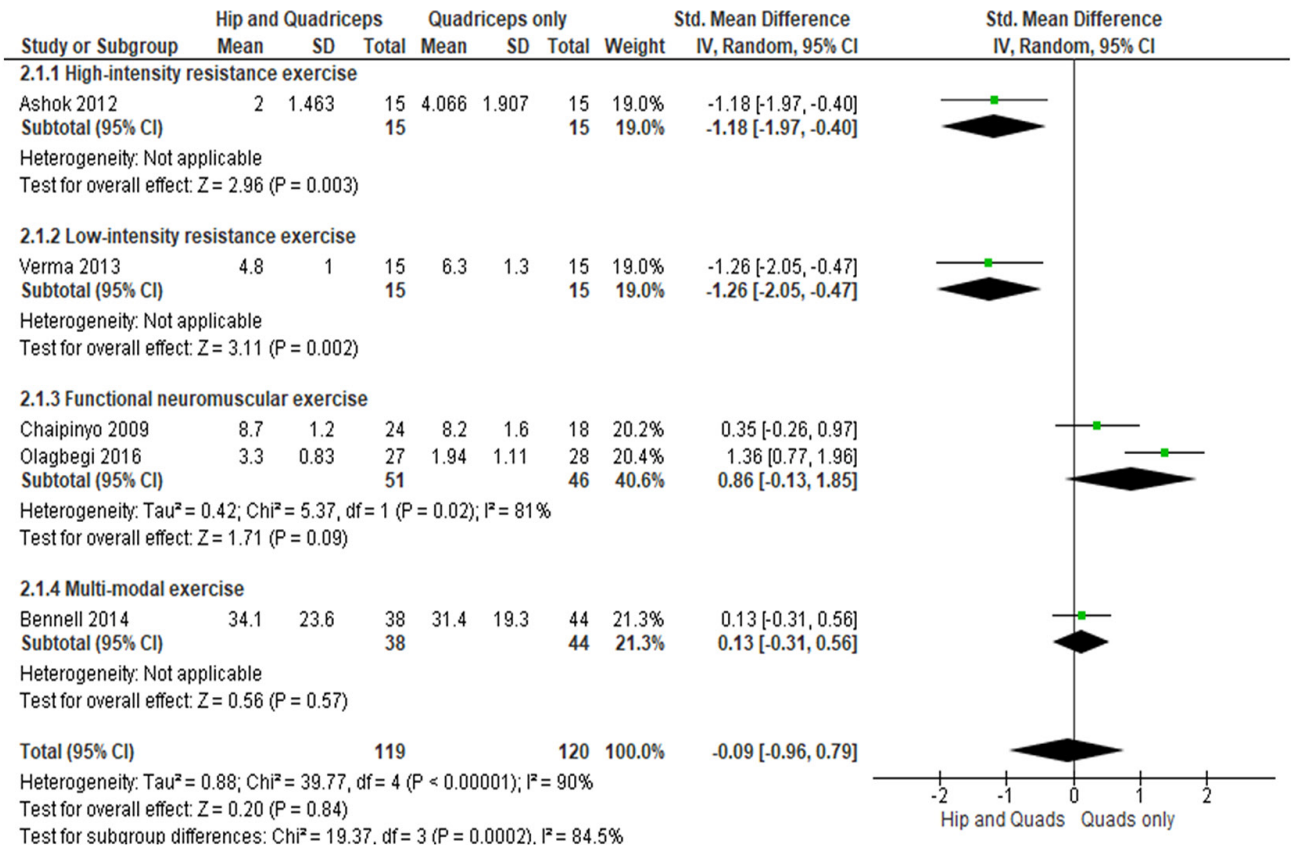

B

\begin{tabular}{|c|c|c|c|c|c|c|c|c|c|}
\hline \multirow[b]{2}{*}{ Study or Subgroup } & \multicolumn{3}{|c|}{ Hip and Quadriceps } & \multicolumn{3}{|c|}{ Quadriceps only } & \multicolumn{2}{|r|}{ Std. Mean Difference } & \multirow{2}{*}{$\begin{array}{l}\text { Std. Mean Difference } \\
\text { IV, Random, } 95 \% \mathrm{CI}\end{array}$} \\
\hline & Mean & SD & Total & Mean & SD & Total & Weight & IV, Random, $95 \% \mathrm{Cl}$ & \\
\hline \multicolumn{9}{|c|}{ 3.1.1 High-intensity resistive exercise } & \\
\hline $\begin{array}{l}\text { Ashok } 2012 \\
\text { Subtotal }(95 \% \mathrm{Cl})\end{array}$ & 27.66 & 4.237 & $\begin{array}{l}15 \\
15\end{array}$ & 37.46 & 6.356 & $\begin{array}{l}15 \\
15\end{array}$ & $\begin{array}{l}18.7 \% \\
18.7 \%\end{array}$ & $\begin{array}{l}-1.77[-2.63,-0.90] \\
-1.77[-2.63,-0.90]\end{array}$ & \\
\hline
\end{tabular}

Heterogeneity. Not applicable

Test for overall effect: $Z=4.02(P<0.0001)$

3.1.2 Low-intensity resistive exercise

$\begin{array}{lrrrrrrrr}\text { Singh } 2016 & 59.46 & 12.44 & 15 & 82.73 & 13.96 & 15 & 18.8 \% & -1.71[-2.57,-0.86]\end{array}$

$\begin{array}{lllllllll}\text { Verma } 2013 & 55.7 & 6.5 & 15 & 61.6 & 7.2 & 15 & 19.7 \% & -0.84[-1.59,-0.09] \\ \text { Subtotal }(95 \% \mathrm{Cl}) & & & 30 & & & 30 & 38.4 \% & -1.25[-2.11,-0.39]\end{array}$

Heterogeneity: Tau $^{2}=0.22 ; \mathrm{Chi}^{2}=2.28, \mathrm{df}=1(P=0.13) ; \mathrm{i}^{2}=56 \%$

Test for overall effect: $Z=2.86(P=0.004)$

3.1.3 Functional neuromuscular exercise

$\begin{array}{lllllllll}\text { Chaipinyo } 2009 & 88 & 10 & 24 & 82 & 13 & 18 & 20.8 \% & 0.52[-0.10,1.14] \\ \text { Subtotal }(95 \% \mathrm{Cl}) & & & 24 & & & \mathbf{1 8} & 20.8 \% & 0.52[-0.10,1.14]\end{array}$

$0.52[-0.10,1.14]$

Heterogeneity: Not applicable

Test for overall effect: $Z=1.63(P=0.10)$

3.1.4 Multi-modal exercise

$\begin{array}{lllllllll}\text { Bennell } 2014 & 28.3 & 14.1 & 38 & 30.4 & 14.5 & 44 & 22.1 \% & -0.15[-0.58,0.29] \\ \text { Subtotal }(95 \% \mathrm{Cl}) & & & 38 & & & 44 & 22.1 \% & -0.15[-0.58,0.29]\end{array}$

Heterogeneity. Not applicable

Test for overall effect: $Z=0.66(P=0.51)$

Total $(95 \% \mathrm{Cl}) \quad 107 \quad 107 \quad 100.0 \% \quad-0.74[-1.56,0.08]$

Heterogeneity Tau $^{2}=0.74 ; \mathrm{Chi}^{2}=29.56, \mathrm{df}=4(P<0.00001) ; \mathrm{F}^{2}=86 \%$

Test for overall effect: $Z=1.78(P=0.08)$

Test for subaroun differences: $\mathrm{Chi}^{2}=22.93 . \mathrm{df}=3(\mathrm{P}<0.0001) . \mathrm{F}^{2}=86.9 \%$

C

Hip and Quadriceps Quadriceps only Std. Mean Difference

Study or Subgroup Mean SD Total Mean SD Total IV, Random, $95 \% \mathrm{Cl}$

\begin{tabular}{lllllllll}
\hline 5.1.1 Functional neuromuscular exercise & & & & & \\
Chaipinyo 2009 & -70 & 14 & 24 & -62 & 23 & 18 & $-0.43[-1.05,0.19]$
\end{tabular}

Chaping $2009-23-18-0.43[-1.05,0.19]$

5.1.2 Multi-modal exercise

$\begin{array}{llllllll}\text { Bennell } 2014 & 0.78 & 0.14 & 38 & 0.78 & 0.18 & 44 & 0.00[-0.43,0.43]\end{array}$

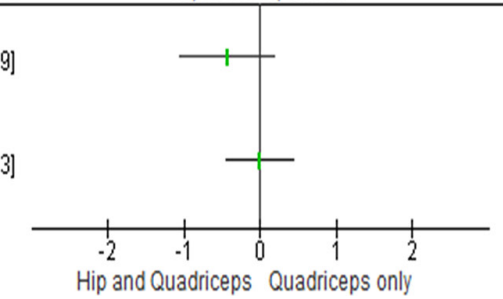

Figure 2 Patient-reported outcomes. (A) Pain, (B) function, (C) quality of life.

favoured the addition of low-intensity hip resistance exercise $(-1.48,95 \% \text { CI }-2.3 \text { to }-0.66)^{22}$ and functional neuromuscular exercises $(-1.5,95 \% \text { CI }-2.03 \text { to }-0.97)^{2324}$ to quadriceps exercises alone. The addition of a multimodal hip exercise programme to quadriceps exercises resulted in no further benefit than quadriceps exercises alone in terms of walking function. ${ }^{19}$ Effect sizes were unable to be calculated for one study, ${ }^{41}$ which reported no benefit of adding neuromuscular hip exercises to quadriceps exercises alone for improving walking function.

\section{Sit to stand}

Sit to stand function was reported in three studies (two highquality $^{1924}$ and one low-quality ${ }^{20}$ ) totalling 178 participants 


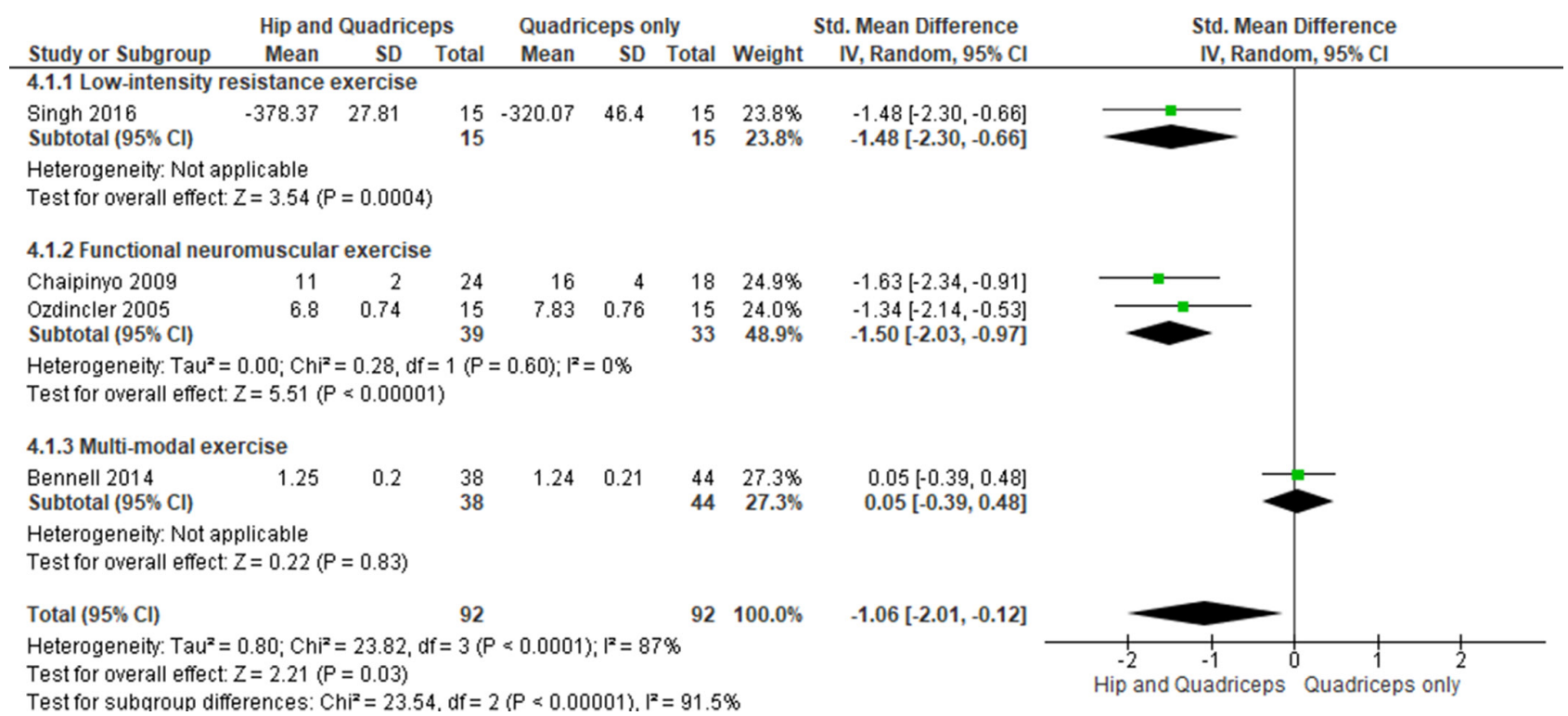

Figure 3 Physical function tests. (A) Walking function, (B) sit to stand, (C) stair negotiation, (D) balance performance.

(figure 3B). Pooled findings demonstrated that combining hip and quadriceps exercises is no better than quadriceps exercises alone for improving sit to stand performance $(-0.85,95 \% \mathrm{CI}$ -1.76 to 0.06$)$. This is consistent with pooled findings for two studies on medial $\mathrm{KOA}^{1920}(-0.47,95 \% \mathrm{CI}-1.25$ to 0.31$)$ (online supplementary file 7 ). The difference between subgroups of types of hip exercise used was significant $(p<0.01)$. From single studies, there is limited evidence of large effect that lowintensity resistance $(-0.93,95 \% \text { CI }-1.69 \text { to }-0.17)^{20}$ and functional neuromuscular hip exercises $(-1.59$, 95\% CI -2.29 to -0.88$)^{24}$ are superior to quadriceps exercise alone for sit to stand function. Data from a single study showed no additional benefit from combining multimodal hip exercise programme with quadriceps exercises on outcomes of sit to stand performance $(-0.13,95 \% \mathrm{CI}-0.56$ to 0.31$) .^{19}$

\section{Stairs}

Stair negotiation was reported in three studies (two highquality $^{1924}$ and one low-quality ${ }^{23}$ ) totalling 178 participants (figure $3 \mathrm{C}$ ). Pooled data from the three studies demonstrated no significant effect of adding hip exercises to quadriceps exercises, compared with quadriceps exercises alone $(-0.79,95 \%$ CI -1.67 to 0.26 ). There were no studies that investigated hip resistance exercises for stair negotiation. There was a significant difference between outcomes for functional neuromuscular exercises and multimodal exercises $(\mathrm{p}<0.001)$. Pooled data from two studies ${ }^{2324}$ showed a significantly large effect favouring the addition of functional neuromuscular exercises to quadriceps on outcomes of stair negotiation $(-1.18,95 \%$ CI -1.68 to -0.67$)$. A single study found no benefit in adding a multimodal hip exercise programme to quadriceps strengthening for stair performance $(0.13,95 \% \mathrm{CI}-0.30$ to 0.57$) .^{19}$

\section{Balance}

Balance step test was reported in two studies (one high-quality ${ }^{19}$ and one low-quality ${ }^{20}$ ) totalling 130 participants (figure $3 \mathrm{D}$ ). Meta-analysis demonstrated a small non-significant effect overall favouring hip and quadriceps exercises over quadriceps alone $(-0.48,95 \%$ CI -1.37 to 0.41$)$. Although the test for subgroup differences did not reach statistical significance $(p=0.07)$, effect sizes reveal differences in outcomes between resistance exercises and multimodal hip exercises. Limited evidence from one study demonstrated that low-intensity resisted hip exercises in combination with quadriceps exercises had a large significant effect when compared with quadriceps alone for balance $(-0.89,95 \%$ CI -1.65 to -0.13$).^{20}$ In comparison, a multimodal hip exercise programme in addition to quadriceps exercises was no more effective than quadriceps exercises alone for improving balance performance $(-0.08,95 \% \mathrm{CI}-0.51$ to 0.36$){ }^{19}$

Quality of meta-analyses (GRADE) are presented in online supplementary file 5. Combining all types of hip strengthening interventions in an overall meta-analysis was graded as very low for all outcomes. When more than one study was available for each subtype of hip strengthening intervention, the quality of evidence ranged from very low to moderate.

Sensitivity analyses of high-quality studies for patientreported outcomes and physical function tests are presented in online supplementary files 8 and 9, respectively. Two highquality studies, one evaluating functional neuromuscular exercises $^{24}$ and one a multimodal programme, ${ }^{19}$ were included in these analyses. Low-quality evidence demonstrated that the addition of hip exercises to quadriceps exercises provided no greater benefit for patient-reported outcomes of pain $(0.20$, $95 \%$ CI -0.15 to 0.56$)$ or function $(0.15,95 \%$ CI -0.5 to 0.79). ${ }^{19} 24$ Very low-quality evidence did not support the addition of these hip exercise programmes to quadriceps for walking $(-0.76,95 \% \mathrm{CI}-2.4$ to 0.88$)$ or sit to stand $(-0.83$, $95 \%$ CI -2.23 to 0.60 ) function. ${ }^{19} 24$

Publication bias was not evaluated due to an insufficient number of studies in each meta-analysis. ${ }^{44}$

\section{DISCUSSION}

The available very low-quality evidence from 8 studies totalling 341 participants with KOA does not support the addition of hip exercises to a quadriceps exercise programme for improving reported pain, function or QoL. Adding hip exercises to a quadriceps exercise programme does have additional benefits for walking. Subgroup analyses of resisted hip and quadriceps exercises consistently resulted in better outcomes than quadriceps exercises alone. Subgroup analyses of people with medial KOA demonstrated superior outcomes in patientreported function when hip and quadriceps exercises were 
combined, compared with quadriceps alone. Sensitivity analysis including high-quality studies reduced confidence in these findings.

Hip exercise type might be related to outcomes. High-intensity and low-intensity hip resistance exercises both demonstrated large significant effects for patient-reported pain and function, compared with quadriceps alone. Low-intensity resistance hip exercises also demonstrated significant large effect sizes for functional performance of walking, sit to stand and balance. No studies examined the effect of resisted hip exercise on stair negotiation or QoL. The addition of functional neuromuscular hip exercises to quadriceps strengthening was beneficial for walking, stairs and sit to stand but not for patient-reported measures of pain (one highquality and one low-quality study), ${ }^{24}{ }^{25}$ function (one high-quality study) ${ }^{24}$ or QoL (one high-quality study). ${ }^{24} \mathrm{~A}$ multimodal hip exercise programme (including both resistance and functional neuromuscular hip exercises) in addition to quadriceps exercises was not superior to quadriceps alone for any outcome.

This systematic review provides evidence that clinicians should consider the type of hip exercises prescribed for people with KOA, to enhance outcomes over quadriceps alone. Hip exercises that included a resistance component as defined in this review appear to have the greatest benefit over quadriceps exercises alone, particularly for improving pain and function in the short term. The benefit of hip resistance exercises in people with KOA is consistent with reports on quadriceps resistance exercises ${ }^{27}$ where the greatest benefits on pain and disability were identified in those who achieved at least $30 \%$ gains in quadriceps strength. ${ }^{9}$

Strengthening the hip muscles, particularly the hip abductors, might improve pelvic drop and trunk control during stance phase. ${ }^{14}$ This has the potential to reduce the knee adduction moment, and has implications for medial knee compartment load. ${ }^{13}$ This may explain the improvement in walking function seen in this study regardless of type of hip exercise employed, as well as greater benefits observed in patient-reported function for those with medial KOA.

Improvements in patient-reported function could be mediated by improvements in strength associated with hip strengthening exercises. One study included in our review, which reported large effects on patient-reported function with low-intensity resisted hip exercises, also reported concurrent improvements in hip abduction strength of $22 \%{ }^{22}$ In comparison, only $9 \%$ gains in hip abduction strength were reported in a study that demonstrated no difference between multi modal hip exercises with quadriceps strengthening, compared with quadriceps exercises alone, for outcomes of patientreported function. ${ }^{19}$ This suggests that greater changes in hip abduction strength may be associated with greater improvements in patient-reported function. Indeed, increased knee extensor strength following a quadriceps strengthening programme has been shown to partially mediate the effect of the exercise programme on pain and physical function in people with $\mathrm{KOA} .{ }^{45}$ We were unable to investigate this association in this systematic review due to the lack of reporting of strength changes over time. ${ }^{8}$ However, it is clear that future clinical trials of hip muscle strengthening should evaluate the concurrent effects on strength, and how changes in strength mediate changes in symptoms, function and QoL.

Interestingly, we found very little difference in outcomes between high-intensity or low-intensity hip resisted exercises. This may be due to a small number of studies and only shortterm follow-up times reported. A previous systematic review ${ }^{8}$ on people with hip or KOA identified that at least moderate gains in strength are achieved in the short term for both lowintensity and high-intensity resistance programmes. This may be sufficient to show relatively equal benefits of low-intensity and high-intensity hip resistance exercises across outcomes in our review (all measured in the short term). The review by Zacharias et $a l^{8}$ also suggests that sustained benefits (up to 6 months) in strength are only achieved by high-intensity resistance exercises. It may be that differences between outcomes of high-intensity and low-intensity resisted hip exercises may only become apparent beyond short-term follow-up. The quality of studies or limited sample may also have contributed to the lack of difference between high-intensity and lowintensity resistance exercises.

Functional neuromuscular hip exercises did not appear to provide added benefit to quadriceps exercises alone, on outcomes of patient-reported pain or function. However, there were benefits for objective measures of function including walking, sit to stand and stair negotiation. The mechanism may be in part due to biomechanics, improving trunk on pelvis or femoro-pelvic alignment in the frontal plane. Functional neuromuscular exercises may also have effects on psychosocial characteristics such as kinesiophobia or self-efficacy, which is task-specific. ${ }^{46}$

Reporting of exercise details is imperative to enable assessment of heterogeneity between studies and replication of interventions. ${ }^{47}$ More than $80 \%$ of the studies in this review reported details of exercises, whether they were individualised and the exercise equipment used. Exercise progression, adherence, motivation strategies, programme fidelity and the qualifications and/or experience of instructors were poorly reported. This needs to be considered when interpreting study outcomes, and in designing future clinical trials. In particular, findings of non-significant differences between hip and quadriceps exercises compared with quadriceps exercises alone may not be due to lack of efficacy, but may reflect differences in adherence. ${ }^{48}$

There are several limitations associated with this review. When grouping all types of hip exercises in addition to quadriceps exercises, the quality of evidence was rated very low (GRADE). Results were inconsistent with some interventions favouring quadriceps only. ${ }^{24}{ }^{25}$ In most circumstances, there were only single studies for analyses of different types of hip intervention and meta-analyses could not be conducted. All follow-up time points were 3 months or less in duration. KOA is a chronic condition, with long-term management strategies needed to prevent progression to surgical intervention. Results are also limited to radiological diagnosis of KOA of mild-tomoderate severity, as only $10 \%$ of participants in this review had severe radiological KOA.

Further research is needed, especially regarding intermediate outcomes to long-term outcomes of hip-focused resistance and neuromuscular functional exercises in KOA. Future studies should investigate the benefits of high-intensity compared with low-intensity resisted hip strength training in addition to high-intensity resisted quadriceps strengthening for patient-reported outcomes. Exercise intervention studies should report on all elements of the CERT, ${ }^{47}$ especially in reporting exercise progressions, adherence, motivation strategies and the qualifications of instructors.

\section{CONCLUSION}

The addition of hip exercises to conventional quadriceps strengthening results in no greater benefit except for walking in the short-term for people with KOA. These effects may be mediated by the type of hip intervention (eg, resistance) and 


\section{What is already known}

- Land-based exercise reduces pain and improves function in people with knee osteoarthritis (KOA) over the short to medium term.

- People with KOA have weakness in hip abduction is $(7 \%$ $24 \%$ weaker than control patients).

- Hip abduction strength is positively related to function in people with KOA.

- Hip strengthening is beneficial compared with non-exercise interventions in people with KOA.

\section{What are the new findings}

- Adding resistance hip exercises to quadriceps exercises is beneficial for patient-reported outcomes and physical function in people with KOA in the short-term.

- There was no difference between high-intensity and lowintensity resistance hip exercises across short-term outcomes.

- Functional neuromuscular hip exercises combined with quadriceps exercises improved physical function (eg, walking) but not patient-reported outcomes.

- Exercise progression, adherence, motivation strategies, programme fidelity and the qualifications and/or experience of instructors is poorly reported.

the location of KOA (eg, medial compartment). The addition of resistance hip exercise to quadriceps strengthening has greater benefits for patient-reported outcomes. For those with medial KOA, the addition of hip resistance exercise has greater benefits on patient-reported function. Based on these findings, clinicians are encouraged to prescribe hip exercise in addition to quadriceps for people with KOA, especially in the presence of medial KOA. The effect of hip exercises in addition to quadriceps and higher-intensity hip resistance over the long term needs to be investigated further.

Contributors The authors confirm that all named authors meet the Uniform Requirements for Manuscripts Submitted to Biomedical Journals criteria for authorship. The authors also confirm that the manuscript has not been submitted or is not simultaneously being submitted elsewhere, and that no portion of the data has been or will be published in proceedings or transactions of meetings or symposium volumes. The authors have no financial disclosures to declare.

Funding The authors have not declared a specific grant for this research from any funding agency in the public, commercial or not-for-profit sectors.

Competing interests None declared.

Patient consent Not required.

Provenance and peer review Not commissioned; externally peer reviewed.

\section{ORCID iDs}

Natalie J Collins http://orcid.org/0000-0001-9950-0192

Adam Ivan Semciw http://orcid.org/0000-0001-5399-7463

\section{REFERENCES}

1 Kittelson AJ, George SZ, Maluf KS, et al. Future directions in painful knee osteoarthritis: harnessing complexity in a heterogeneous population. Phys Ther 2014;94:422-32.

2 Fransen M, McConnell S, Harmer AR, et al. Exercise for osteoarthritis of the knee. Cochrane Database Syst Rev 2015;1:CD004376.

3 Richmond J, Hunter D, Irrgang J, et al. Treatment of osteoarthritis of the knee (nonarthroplasty). J Am Acad Orthop Surg 2009;17:591-600.

4 Allen KD, Golightly YM. State of the evidence. Curr Opin Rheumatol 2015;27:276-83

5 Farr li J, Miller LE, Block JE. Quality of life in patients with knee osteoarthritis: a commentary on nonsurgical and surgical treatments. Open Orthop J 2013;7:619-23.
6 Cross M, Smith E, Hoy D, et al. The global burden of hip and knee osteoarthritis: estimates from the global burden of disease 2010 study. Ann Rheum Dis 2014;73:1323-30.

7 McAlindon TE, Bannuru RR, Sullivan MC, et al. OARSI guidelines for the non-surgical management of knee osteoarthritis. Osteoarthritis Cartilage 2014;22:363-88.

8 Zacharias A, Green RA, Semciw Al, et al. Efficacy of rehabilitation programs for improving muscle strength in people with hip or knee osteoarthritis: a systematic review with meta-analysis. Osteoarthritis Cartilage 2014;22:1752-73.

9 Bartholdy C, Juhl C, Christensen R, et al. The role of muscle strengthening in exercise therapy for knee osteoarthritis: a systematic review and meta-regression analysis of randomized trials. Semin Arthritis Rheum 2017;47:9-21

10 Deasy M, Leahy E, Semciw Al. Hip Strength deficits in people with symptomatic knee osteoarthritis: a systematic review with meta-analysis. J Orthop Sports Phys Ther 2016;46:629-39

11 Tevald MA, Murray A, Luc BA, et al. Hip abductor strength in people with knee osteoarthritis: a cross-sectional study of reliability and association with function. Knee 2016;23:57-62.

12 Park SK, Kobsar D, Ferber R. Relationship between lower limb muscle strength, selfreported pain and function, and frontal plane gait kinematics in knee osteoarthritis. Clin Biomech 2016;38:68-74.

13 Dunphy C, Casey S, Lomond A, et al. Contralateral pelvic drop during gait increases knee adduction moments of asymptomatic individuals. Hum Mov Sci 2016;49:27-35.

14 Mündermann A, Dyrby CO, Andriacchi TP. Secondary gait changes in patients with medial compartment knee osteoarthritis: increased load at the ankle, knee, and hip during walking. Arthritis Rheum 2005;52:2835-44.

15 Chang A, Hayes K, Dunlop D, et al. Hip abduction moment and protection against medial tibiofemoral osteoarthritis progression. Arthritis Rheum 2005;52:3515-9.

16 Brosseau L, Taki J, Desjardins B, et al. The Ottawa panel clinical practice guidelines for the management of knee osteoarthritis. Part two: strengthening exercise programs. Clin Rehabil 2017;31:596-611.

17 Bennell KL, Hunt MA, Wrigley TV, et al. Hip strengthening reduces symptoms but not knee load in people with medial knee osteoarthritis and varus malalignment: a randomised controlled trial. Osteoarthritis Cartilage 2010;18:621-8.

18 Jorge RT, Souza MC, Chiari A, et al. Progressive resistance exercise in women with osteoarthritis of the knee: a randomized controlled trial. Clin Rehabil 2015;29:234-43.

19 Bennell KL, Kyriakides M, Metcalf B, et al. Neuromuscular versus quadriceps strengthening exercise in patients with medial knee osteoarthritis and varus malalignment: a randomized controlled trial. Arthritis Rheumatol 2014:66:950-9.

20 Verma S, Agarwal S. The effect of hip abductors strengthening exercise on knee pain and function in people with knee osteoarthritis. Sports Medicine Journal / Medicina Sportivâ 2013;9:2123-9.

21 Ashok C. Effects of hip abductor muscle strengthening exercises in patients with osteoarthritic knee joints. Indian Journal of Physiotherapy \& Occupational Therapy 2012;6:32-7.

22 Singh S, Pattnaik M, Mohanty $P$, et al. Effectiveness of hip abductor strengthening on health status, strength, endurance and six minute walk test in participants with medial compartment symptomatic knee osteoarthritis. J Back Musculoskelet Rehabil 2016;29:65-75.

23 Özdinçler AR, Yeldan I, Kinali P. The effects of closed kinetic chain exercise on pain and functional performance of patients with knee osteoarthritis. The Pain Clinic 2005; 17:107-15.

24 Chaipinyo K, Karoonsupcharoen 0 . No difference between home-based strength training and home-based balance training on pain in patients with knee osteoarthritis: a randomised trial. Aust J Physiother 2009;55:25-30.

25 Olagbegi OM, Adegoke BOA, Odole AC. Effectiveness of combined chain exercises on pain and function in patients with knee osteoarthritis. Bangladesh Journal of Medical Science 2016;15:178-88.

26 Crossley KM, Zhang WJ, Schache AG, et al. Performance on the single-leg squat task indicates hip abductor muscle function. Am J Sports Med 2011;39:866-73.

27 Fransen M, McConnell S, Harmer AR, et al. Exercise for osteoarthritis of the knee: a Cochrane systematic review. Br J Sports Med 2015;49:1554-7.

28 Jüni P, Reichenbach S, Dieppe P. Osteoarthritis: rational approach to treating the individual. Best Pract Res Clin Rheumatol 2006;20:721-40.

29 Dobson F, Hinman RS, Roos EM, et al. OARSI recommended performance-based tests to assess physical function in people diagnosed with hip or knee osteoarthritis. Osteoarthritis Cartilage 2013;21:1042-52.

30 Adegoke BOA, Boyinde $\mathrm{OH}$, Odole AC, et al. Do self-efficacy, body mass index, duration of onset and pain intensity determine performance on selected physical tasks in individuals with unilateral knee osteoarthritis? Musculoskelet Sci Pract 2017;32:1-6.

31 Slade S, Mastwyk S, Morris M, et al. Optimising uptake and implementation of pelvic floor muscle training exercise programs for people with urinary incontinence: a qualitative study. Australian \& New Zealand Continence Journal 2017:23:101-2.

32 Wan X, Wang W, Liu J, et al. Estimating the sample mean and standard deviation from the sample size, median, range and/or interquartile range. BMC Med Res Methodol 2014; $14: 135-35$ 
33 Maher CG, Sherrington C, Herbert RD, et al. Reliability of the PEDro scale for rating quality of randomized controlled trials. Phys Ther 2003;83:713-21.

34 de Morton NA. The PEDro scale is a valid measure of the methodological quality of clinical trials: a demographic study. Aust J Physiother 2009;55:129-33.

35 Moher D, Jadad AR, Tugwell P. Assessing the quality of randomized controlled trials. Current issues and future directions. Int I Technol Assess Health Care 1996;12:195-208

36 Lack S, Barton C, Sohan O, et al. Proximal muscle rehabilitation is effective for patellofemoral pain: a systematic review with meta-analysis. Br I Sports Med 2015:49:1365-76.

37 Cohen J. A power primer. Psychol Bull 1992;112:155-9

38 Higgins JP, Thompson SG, Deeks JJ, et al. Measuring inconsistency in meta-analyses. BMJ 2003;327:557-60.

39 Guyatt GH, Oxman AD, Kunz R, et al. What is "quality of evidence" and why is it important to clinicians? BMJ 2008:336:995-8.

40 Balshem $\mathrm{H}$, Helfand M, Schünemann HJ, et al. GRADE guidelines: 3 . Rating the quality of evidence. J Clin Epidemiol 2011:64:401-6.

41 Callaghan MJ, Oldham JA, Hunt J. An evaluation of exercise regimes for patients with osteoarthritis of the knee: a single-blind randomized controlled trial. Clin Rehabil 1995;9:213-8.
42 Dobson F, Hinman RS, Hall M, et al. Reliability and measurement error of the Osteoarthritis Research Society International (OARSI) recommended performancebased tests of physical function in people with hip and knee osteoarthritis. Osteoarthritis Cartilage 2017:25:1792-6.

43 Filbay SR, Culvenor AG, Ackerman IN, et al. Quality of life in anterior cruciate ligament-deficient individuals: a systematic review and meta-analysis. Br J Sports Med 2015;49:1033-41.

44 Lau J, loannidis JP, Terrin N, et al. The case of the misleading funnel plot. BMJ 2006;333:597-600.

45 Hall M, Hinman RS, Wrigley TV, et al. Knee extensor strength gains mediate symptom improvement in knee osteoarthritis: secondary analysis of a randomised controlled trial. Osteoarthritis Cartilage 2018;26:495-500.

46 Rejeski WJ, Ettinger WH, Martin K, et al. Treating disability in knee osteoarthritis with exercise therapy: a central role for self-efficacy and pain. Arthritis Care Res 1998;11:94-101.

47 Slade SC, Dionne CE, Underwood M, et al. Consensus on Exercise Reporting Template (CERT): modified Delphi Study. Phys Ther 2016;96:1514-24.

48 Minshull C, Gleeson N. Considerations of the principles of resistance training in exercise studies for the management of knee osteoarthritis: a systematic review. Arch Phys Med Rehabil 2017;98:1842-51. 